\title{
The Analysis of Interplanetary Shocks Associated with Six Major Geo-Effective Coronal Mass Ejections during Solar Cycle 24
}

\author{
Shirsh Lata Soni1 ${ }^{*}$, Prithvi Raj Singh², Bharti Nigam1, Radhe Syam Gupta1, \\ Pankaj Kumar Shrivastava ${ }^{3}$
}

${ }^{1}$ Department of Physics, Govt. P.G. College, Satna, India

${ }^{2}$ Department of Physics, APS University, Rewa, India

${ }^{3}$ Department of Physics, Govt. P.G. Model Science College, Rewa, India

Email: *sheersh171@gmail.com

How to cite this paper: Soni, S.L., Singh, P.R., Nigam, B., Gupta, R.S. and Shrivastava, P.K. (2019) The Analysis of Interplanetary Shocks Associated with Six Major Geo-Effective Coronal Mass Ejections during Solar Cycle 24. International Journal of Astronomy and Astrophysics, 9, 191-199. https://doi.org/10.4236/ijaa.2019.93014

Received: May 9, 2019

Accepted: August 27, 2019

Published: August 30, 2019

Copyright $\odot 2019$ by author(s) and Scientific Research Publishing Inc. This work is licensed under the Creative Commons Attribution International License (CC BY 4.0).

http://creativecommons.org/licenses/by/4.0/

\begin{abstract}
A Coronal Mass Ejection (CME) is an ejection of energetic plasma with magnetic field from the Sun. In traversing the Sun-Earth distance, the kinematics of the CME is immensely important for the prediction of space weather. The objective of the present work is to study the propagation properties of six major geo-effective CMEs and their associated interplanetary shocks which were observed during solar cycle 24 . These reported CME events produced intense geo-magnetic storms (Dst $>140 \mathrm{nT}$ ). The six CME events have a broad range of initial linear speeds $\sim 600-2700 \mathrm{~km} / \mathrm{sec}$ in the LASCO/SOHO field of view, comparing two slow CMEs (speed $\sim 579 \mathrm{~km} / \mathrm{sec}$ and $719 \mathrm{~km} / \mathrm{sec}$ ), three moderate speed CMEs (speed $\sim 1366,1571,1008$ $\mathrm{km} / \mathrm{sec}$ ), and one fast CME (speed $\sim 2684 \mathrm{~km} / \mathrm{sec}$ ). The actual arrival time of the reported events is compared with the arrival time calculated using the Empirical Shock Arrival model (ESA model). For acceleration estimation, we utilize three different acceleration-speed equations reported in the previous literatures for different acceleration cessation distance (ACD). In addition, we compared the transit time estimated using the second-order speed of CMEs with observed transit time. We also compared the observed transit time with transit time obtained from various shock arrival model. From our present study, we found the importance of acceleration cessation distance for CME propagation in interplanetary space and better acceleration speed for transit time calculation than other equations for CME forecasting.
\end{abstract}

\section{Keywords}

Coronal Mass Ejection (CME), IP Shock, Geomagnetic Strom 


\section{Introduction}

Coronal Mass Ejection is the most energetic process of solar atmosphere. The CME can be defined as an ejection of plasma with magnetic field from the Sun to the interplanetary space. And its effect on Earth's environment and space weather. Kinematics of CMEs in space depends upon the initial seed additionally affected by ambient solar wind conditions [1] [2] [3]. The propagation of coronal mass ejections has variations continuously due to their internal energy and interaction with other into interplanetary [4] [5]. The Earth directed CME (i.e. halo or partial halo CME) affect the magnetosphere of Earth. These CME knows as geo-effective CME, the geo-effectiveness of CME identified by geo-magnetic storm disturbances index; Dst (or horizontal component of geo-magnetic disturbance field; SYM/H index). The geo-effectiveness is high, if the value of Dst is more negative. In the interplanetary medium, the CME went through acceleration and deceleration due to solar wind speed and finally come to speed nearly equal to speed of solar wind [6] [7]. But as we know that the speed of solar wind shows variation during the 11 year period of solar cycle. Estimation of the arrival of CME to near Earth is very important for predicting the space weather. There is no certain method for model to calculate the arrival time of CME at $1 \mathrm{AU}$ accurately. However, Gopalswamy, in 2001, estimated the transit time of 47 Earth directed CME events observed during the period of 1996 to 2000 following the Empirical shock arrival (ESA) model. For estimation of transit time, they proposed a formula for acceleration $(a=2.193-0.0054 u)$ related to the initial speed (u) of CMEs [7]. While the transit time for 83 halo CME events at 1 AU investigated by Michalek et al. 2004 [8]. Among these 83 events, an equation was obtained between effective acceleration and initial speed as $a=4.11-0.0063 u$ for 49 CME events with several fast. Another acceleration equation $a=3.35-$ $0.0074 u$, was obtained for extreme events including very fast CMEs ( 2684 $\mathrm{km} / \mathrm{sec})$, few very slow speed CMEs $(\sim 400 \mathrm{~km} / \mathrm{sec})$ and two main cases were chosen to representing events for which: 1) there is no acceleration of CMEs and 2) accelerating CMEs at $1 \mathrm{AU}$. In the present work, we examine the propagation of six geo-effective CME events having a wide range of linear initial speed ( 600 to $2700 \mathrm{~km} / \mathrm{sec}$ ) and produced intense geo-magnetic storms having value of Dst index more than $(-140 \mathrm{nT})$. We compare the estimated arrival times with the actual arrival times and also transit time obtained using Drag Based Model (DBM) Vrsnak et al., 2013 [2].

\section{Data Selection}

We studied the set of six major geo-effective CME events observed by SOHO/ LASCO during the solar cycle 24 . The six CME events generated geo-magnetic storms of high intensity Dst $>140 \mathrm{nT}$. These selected CMEs events are associated with C, M and X class X-ray flares. The detail of selected CMEs, associated flare and geo-magnetic storms are listed in Table 1. Geo-effective CME details are also obtained from the online catalogues of SOHO/LASCO at https://cdaw.gsfc.nasa.gov/CME_list, 
Table 1. Details for major geo-effective CMEs with associated flare. Last column shows the detail of generated interplanetary shock with DST value.

\begin{tabular}{|c|c|c|c|c|c|c|c|}
\hline \multirow{2}{*}{$\begin{array}{c}\text { CME } \\
\text { Date/Time }\end{array}$} & \multirow{2}{*}{$\begin{array}{l}\text { Speed } \\
(\mathrm{km} / \mathrm{s})\end{array}$} & \multicolumn{3}{|c|}{ Flare } & \multicolumn{3}{|c|}{ IP Shock } \\
\hline & & $\mathrm{T}_{\text {Rise }} \mathrm{T}_{\text {Peak }} \mathrm{T}_{\text {End }}$ & Intensity & Location & Date/Time & Dst & IP Shock TT \\
\hline $22-10-2011$ 10:25 & 1005 & $15: 14 \quad 15: 2915: 20$ & M1.3 & N29W91 & $24-10-2011$ 18:31 & -147 & 63.17 \\
\hline 07-03-2012 00:24 & 2684 & 00:02 00:40 00:24 & X5.4 & N17E15 & 08-03-2012 11:03 & -145 & 33.4 \\
\hline 15-03-2015 01:48 & 719 & 01:15 03:20 02:13 & C1.3 & S19W25 & 17-03-2015 04:45 & -223 & 50.95 \\
\hline 21-06-2015 02:36 & 1366 & 01:02 02:00 01:42 & M2.0 & N12E16 & $22-06-2015$ 18:33 & -204 & 39.59 \\
\hline 16-12-2015 09:24 & 579 & 08:34 09:23 09:03 & C6.6 & S13W04 & 19-12-2015 16:16 & -155 & 78.86 \\
\hline 06-09-2017 12:24 & 1571 & 11:53 12:10 12:02 & X9.3 & S09W42 & 07-09-2017 22:38 & -142 & 34.23 \\
\hline
\end{tabular}

http://www.lesia.obspm.fr/cesra/highlights/highlight07-5.html. The geomagnetic storms details are obtained from:

http://wdc.kugi.kyoto.u.ac.jp/Dst_realtime/index.html. And Omni web from: https://omniweb.gsfc.nasa.gov/form/dx1.html. X-ray solar flare data (start time, peak time, last time and intensity) obtained from:

https://www.ngdc.noaa.gov/stp/space-weather/solar-data/solar-features/solar-fla res/x-rays/goes/xrs/. The arrival time of CMEs and their associated interplanetary shocks/ICME are determined from the time difference between the first detection (appearance) time of a CME in $\mathrm{C} 2$ coronagraph on-board at SOHO/LASCO and IP shock/ ICME arrival time in ACE/Wind, identified by variation in solar plasma parameters (density, temperature, velocity, etc.) and magnetic field strength at $1 \mathrm{AU}$.

\section{Results and Discussion}

Gopalswamy et al., 2001 and Kim et al., 2007 described a procedure to estimate transit time of CME at $1 \mathrm{AU}$ [7] [9]. The total transit time is given by $T=T_{1}+T_{2}$ where $T_{1}$ is the time of travel up to the acceleration cessation distance $d_{1}$ up to 1 $\mathrm{AU}$ (in Equation (1)) and $T_{2}$ is travel time for reaming distance $d_{2}$ at the constant speed (in Equation (2)). For estimating acceleration, we are using three different equations (Equations (3)-(5)) given by Gopalswamy 2001, Michalek et al. 2004. To obtain the effective interplanetary acceleration from the linear initial speed of CMEs and arrival time with three acceleration cessation distances ( $\mathrm{ACD}=0.7 \mathrm{AU}, 0.6 \mathrm{AU}$ and $0.5 \mathrm{AU}$ ) and then $\mathrm{CME}$ travels the remaining distance (0.3 AU, 0.4 AU and 0.5 AU) with constant speed respectively. Therefore, it is good for study to compare the calculated travel time of these CMEs with the observed travel time. The transit time equations for $T_{1}$ and $T_{2}$ are given by Gopalswamy et al., 2001 are the following:

$$
\begin{gathered}
T_{1}=\frac{-u+\sqrt{\left(u^{2}+2 a d_{1}\right)}}{a} \\
T^{2}=\frac{d_{2}}{\sqrt{\left(u^{2}+2 a d_{1}\right)}}
\end{gathered}
$$


where $u$ is the linear speed and $a$ is the acceleration at interplanetary medium. We calculate interplanetary acceleration of selected CMEs using with different following equations,

$$
\begin{aligned}
& \text { Gopalswamy et al. (2001) } a=2.193-(0.0054 * u) \\
& \text { Michalek et al. (2004) } a=4.11-(0.0063 * u) \\
& \text { Michalek et al. (2004) } a=3.35-(0.007 * u)
\end{aligned}
$$

In the equations above, $u$ is the initial speed of CME. Now we compare the transit times calculated using these above equations with the actual transit time of reported CME events.

\subsection{ESA Model: Arrival Time Using Linear Speed of CMEs}

As we have three equation for calculating transit time, so firstly we calculate Transit time using Equations (1) and (2) with acceleration speed Equation (3) given by Gopalswamy et al.; 2001 at different acceleration cessation distances (0.7 AU, 0.6 AU and 0.5 AU). The graphical representation of transit time for various $\mathrm{ACD} 0.7 \mathrm{AU}, 0.6 \mathrm{AU}$ and $0.5 \mathrm{AU}$ against $\mathrm{CME}$ speed is plotted in Figures 1 (a)-(c) respectively. For comparison, we also plotted transit time profiles estimated by using ESA model for three different acceleration cessation distances. The differences between estimated transit time for various acceleration cessation distances and actual arrival time are reported in Table 2. The first column indicates the sequence of selected CME events as mentioned in Table 1. And column second represents the difference between actual transit time and estimated transit time results for different ACD using Equation (3). From Table 2, it seems that the error in transit time value if less ( $0.3-4$ hour) for event 4 th and 6th, while CME events 1st, 4th and 6th have less error for $0.7 \mathrm{AU}$. This transit time estimation method is repeated for other acceleration-speed equations i.e. (4) and (5) given by Michalek et al. (2004). In the table, columns third and fourth present the difference between actual arrival time and the arrival time

Table 2. Difference between actual transit time with various acceleration cessation distances (0.7 AU, 0.6 AU and 0.5 AU) for Equations (3)-(5). The blue shaded values show the minimum estimated transit time deviation $(\sim 0.6-10 \mathrm{hr})$ from actual transit time.

\begin{tabular}{|c|c|c|c|c|c|c|c|c|c|}
\hline \multirow{2}{*}{$\begin{array}{l}\text { No. of } \\
\text { Event }\end{array}$} & $\Delta \mathrm{T}_{\mathrm{IP} \text { Shock }}$ & \multicolumn{3}{|c|}{ Using Equation (3) $\Delta \mathrm{T}_{\text {IP Shock }}$} & \multicolumn{5}{|c|}{ Using Equation (4) $\Delta \mathrm{T}_{\mathrm{IP} \text { Shock }}$ Using Equation (5) } \\
\hline & $0.7 \mathrm{AU}$ & $0.6 \mathrm{AU}$ & $0.5 \mathrm{AU}$ & $0.7 \mathrm{AU}$ & $0.6 \mathrm{AU}$ & $0.5 \mathrm{AU}$ & $0.7 \mathrm{AU}$ & $0.6 \mathrm{AU}$ & $0.5 \mathrm{AU}$ \\
\hline 1 & 0.76 & 7.57 & 10.324 & 4.77 & 13.84 & 22.186 & 12.033 & 1.51 & 6.323 \\
\hline 2 & 15.29 & 15.49 & 15.792 & 15.18 & 15.39 & 15.72 & 14.41 & 14.75 & 15.19 \\
\hline 3 & -31.75 & -27.22 & -23.44 & -17.03 & 1.529 & -36.51 & -31.54 & -27.39 & -23.34 \\
\hline 4 & -0.38 & 0.893 & -0.38 & 0.791 & 2.568 & 1.733 & -4.484 & -2.09 & 0.18 \\
\hline 5 & 48.46 & 46.33 & 45.21 & 50.3 & 47.651 & 42.88 & 40.46 & 46.349 & 43.68 \\
\hline 6 & 0.61 & 1.48 & 6.4 & 1.51 & 3.64 & 3.07 & -2.42 & -0.83 & 0.79 \\
\hline
\end{tabular}
$\left(\Delta \mathrm{T}_{\text {IP Shock }}=\right.$ difference between actual transit time with transit time obtained from various acceleration-speed equations). 


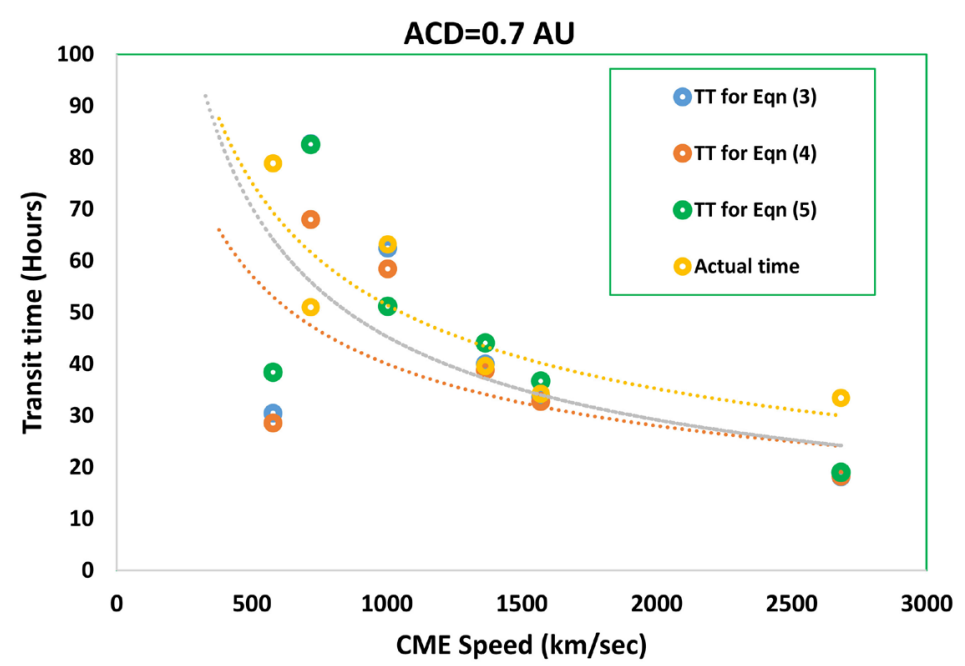

(a)

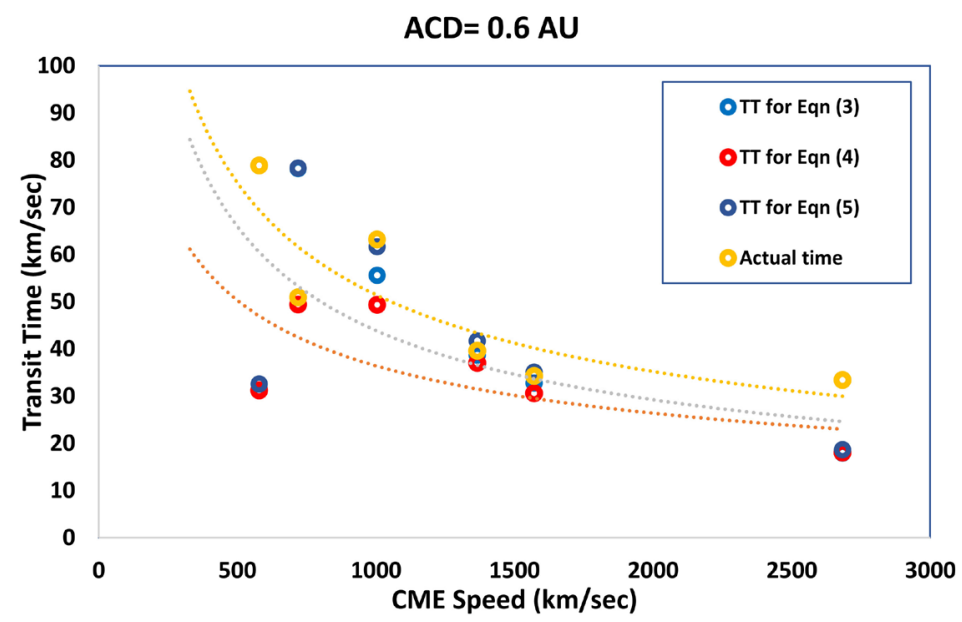

(b)

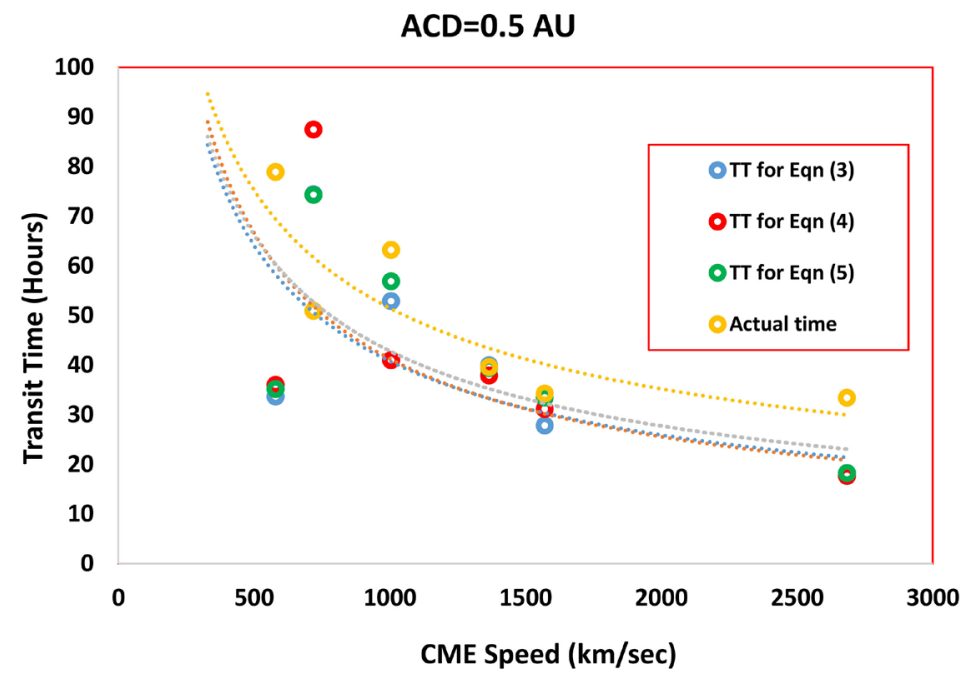

(c)

Figure 1. Comparison between actual transit time (TT) of IP shock and estimated transit time for various acceleration cessation distances (0.7 AU, 0.6 AU and 0.5 AU) obtained from different acceleration-speed Equations (3)-(5) in plot (a), (b) and (c) respectively. 
calculated by using Equations (4) and (5) respectively. On the other hand acceleration-speed Equation (5) gives the approximately nearly consistent transit time value with the actual transit time (approximately $0.8-15 \mathrm{hr}$ ) 6th except event 3rd and 5th events. From the table, we can see that Equation (3) produced the minimum error $\left(\Delta \mathrm{T}_{\mathrm{IP} \text { Shock }}=0.6-10 \mathrm{hr}\right)$ for three CME events (1st, 4th and 6th). We get the actual arrival time values of IP shock from Equation (3) for event 1st, 2nd, 4th and 6th but the deviation is more for other two events (event $3 \mathrm{rd}$ and 5 th), this is may be due to the their slow linear speed $(719 \mathrm{~km} / \mathrm{sec}$ and $579 \mathrm{~km} / \mathrm{sec}$ respectively).

\subsection{ESA Model: Transit Using Second Order Speed of CMEs}

In this section of observation, we obtained the arrival time using second-order speed of CMEs instead of linear speed reported in the LASCO/SOHO catalogue. So here we are estimating transit time from Equation (3) by using two different second-order speed: 1) at 20 solar radius and 2) at final distance at different acceleration cessation distance (0.7 AU) using ESA model with acceleration-speed Equation (3). In Figure 2(a) and Figure 2(b) shows the transit times calculated

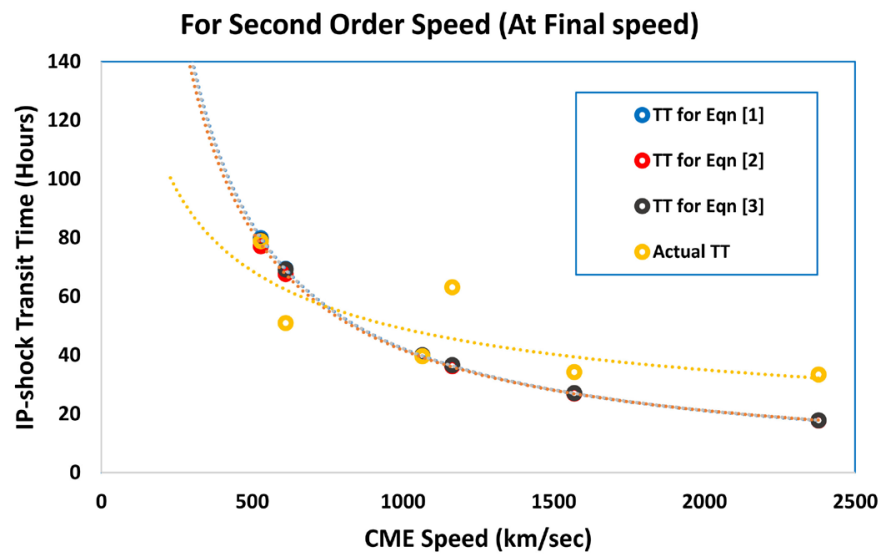

(a)

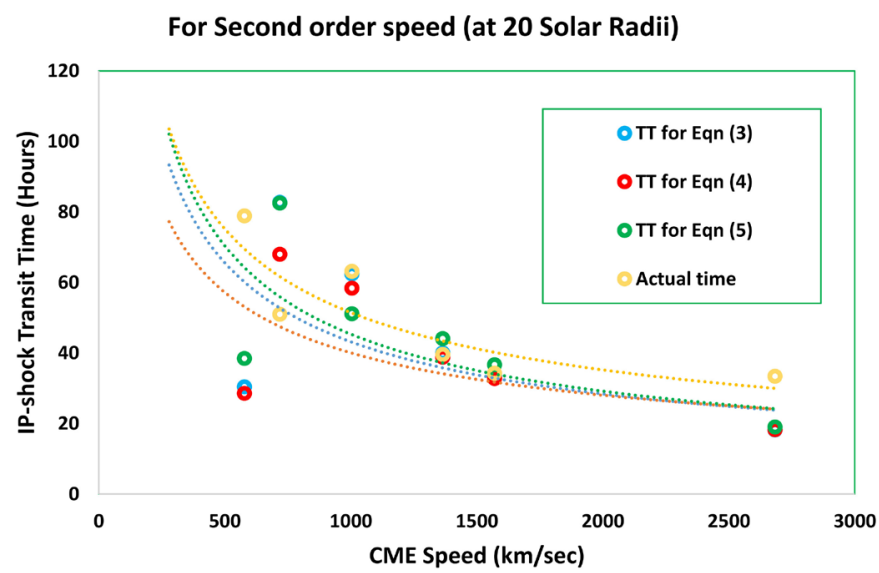

(b)

Figure 2. Transit time calculated using second-order speed (a) at final distance (b) at 20 solar radii. 
using second order speed at 20 solar radius and at final distance respectively. The transit time error (within $\pm 11 \mathrm{hr}$ ) for the last four events, while the transit time error $(\sim 0.2-7 \mathrm{hr})$ for last three events using second-order speed at a final distance. It seems from the investigation above that the speed at final speed gives the arrival time nearly consistent with the actual arrival time.

\subsection{Comparison with Other Models}

In this section, we compare the actual arrival time with transit time obtained by other models (Table 3). Here we are using three different shock arrival prediction models (1) constant Speed Model, (2) Drag Based Model (DBM) proposed by Vrsnak et al. (2013) and (3) transit time prediction model given by Schwenn et al. (2005). The arrival time error for IP shock obtained from the models above with actual transit time are listed in Table 4. In the constant speed model, it has been predicted that CME travels the entire Sun Earth distance at the same speed (initial speed of CME) to reach at $1 \mathrm{AU}$. And also plot these different obtain transit time of IP-shocks in Figure 3. The Drag Based Model assumes that the CME speed dragged due to interaction of ICME and ambient solar wind. In DBM, the given parameters are: starting radial distance of CME $\left(r_{0}\right)$, CME speed at $r_{0}\left(v_{0}\right)$, asymptotic solar wind and drag parameter. The DBM tool is accessible in the website http://oh.geof.unizg.hr/index.php/en/spaceweather-tools. After putting all the values for reported CMEs, we have got the transit times at $1 \mathrm{AU}$. We have taken average ambient solar wind speed as $500 \mathrm{~km} / \mathrm{sec}$, which is the average speed of plasma flow recorded by in-situ instrument. Schwenn et al. (2005) proposed a relationship between the arrival time and linear speed of CMEs as:

$$
T_{r r}=203-20.77 * \ln \left(V_{\mathrm{CME}}\right)
$$

where $T_{r r}$ is arrival time and $V_{\mathrm{CME}}$ is linear speed of CME. In this case of arrival time of interplanetary shock, minimum transit time error is given by DBM model for four events (less than $6 \mathrm{hr}$ ).

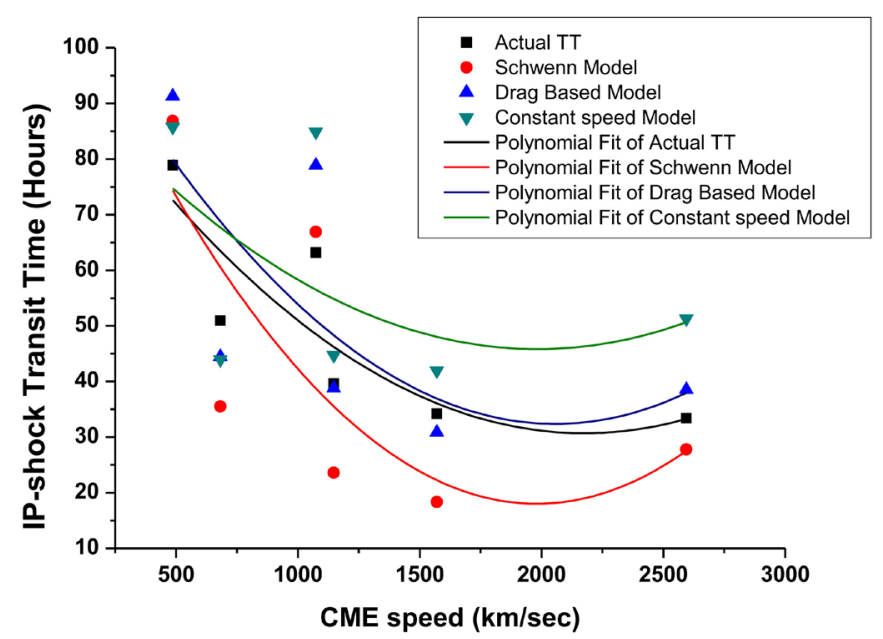

Figure 3. Comparison between observed (actual) transit time and calculated transit time using different models (Schwenn Model, Drag Based Model, Constant Speed Model). 
Table 3. Transit time error for second order speed at final distances in second column and second order speed at 20 solar radii for all three reported CME events.

\begin{tabular}{ccccccc}
\hline \multirow{2}{*}{ No. of Event } & \multicolumn{4}{c}{ Second-Order Speed (at Final Distance) } & \multicolumn{4}{c}{ Second-Order Speed (at 20 Solar Radii) } \\
\cline { 2 - 7 } & $0.7 \mathrm{AU}$ & $0.6 \mathrm{AU}$ & $0.5 \mathrm{AU}$ & $0.7 \mathrm{AU}$ & $0.6 \mathrm{AU}$ & $0.5 \mathrm{AU}$ \\
\hline 1 & 26.73 & 26.67 & 26.75 & 23.64 & 23.58 & 23.67 \\
2 & 15.71 & 15.67 & 15.70 & 17.19 & 17.16 & 17.18 \\
3 & -18.44 & -18.56 & -18.42 & -11.33 & -11.43 & -11.28 \\
4 & -0.27 & -0.34 & -0.25 & 2.60 & 2.54 & 2.62 \\
5 & -0.95 & -1.12 & -0.98 & -7.51 & -7.71 & -7.60 \\
6 & 7.29 & 7.23 & 7.28 & 7.30 & 7.25 & 7.30 \\
\hline
\end{tabular}

Table 4. Difference between the actual transit time and estimated transit time with various models (transit time error) for of IP shocks associated with selected CMEs.

\begin{tabular}{ccc}
\hline \multicolumn{3}{c}{$\Delta \mathrm{T}_{\text {IP Shock }}$ (in Hours) } \\
\hline Schwenn Model & Drag Based Model & Constant Speed Model \\
\hline 3.75 & 15.7 & 21.71 \\
-5.61 & 5.14 & 17.87 \\
-15.42 & -6.47 & -7 \\
-15.99 & -0.79 & 5.12 \\
7.99 & 12.44 & 6.89 \\
-15.91 & -3.34 & 7.7 \\
\hline
\end{tabular}

\section{Conclusion}

For the present study, we presented the estimation of arrival time of six major coronal mass ejections which produced intense geo-magnetic storms more than Dst $>140 \mathrm{nT}$ observed during solar cycle 24 . The reported CME events have different linear speed such as $\sim 579-2684 \mathrm{~km} / \mathrm{sec}$. The interplanetary acceleration values, calculated from the speed of CME using various acceleration-speed equations, are utilized in the ESA model. All the plots regarding transit time of CMEs at $1 \mathrm{AU}$ obtained by using ESA model for three different acceleration cessation distance (0.5 AU, 0.6AU and 0.7AU). Study demonstrated that each event acts differently in the interplanetary space. The CME propagation is also governed by the speed of CME, interplanetary acceleration/deceleration and acceleration cessation distances. Summarizing the above study and analysis, it is seen that the result of all comparison of arrival times for reported six CME, the arrival time (transit time) error is minimum for the acceleration Equation (3) for ESA model. In addition, the transit time are compared with various models (constant speed model, Schwenn Model and Drag Based Model). Especially, the minimum arrival time error is obtained for Drag Based Model (DBM) for the acceleration-speed Equation (4) for ESA model. Presented study also shown that the linear speed provide minimum transit time error, instead of second order speed at final dis- 
tances or at 20 solar radii.

\section{Acknowledgements}

We are grateful to Solar Geo-physical Data team, Kyoto and OMNI data team for their open data source policy. Authors are thankful to SOHO/LASCO CME catalogue (generated and maintained by CDAW data centre by NASA). We thank Prof. Bhuwan Joshi, USO, Physical Research Laboratory, Ahmedabad, and Pro. Hari Om Vats, scientist, Physical Research Laboratory, Ahmedabad India for his great support to us.

\section{Conflicts of Interest}

The authors declare no conflicts of interest regarding the publication of this paper.

\section{References}

[1] Vrsnak, B. and Gopalswamy, N. (2002) Influence of the Acceleration Drag on the Motion of Interplanetary Ejectas. Journal of Geophysical Research: Space Physics, 107, 1009. https://doi.org/10.1029/2001JA000120

[2] Vrsnak, B., Žic, T., Vrbanec, D., Temmer, M., Rollett, T., Möstl, C., Veronig, A., Čalogović, J., Dumbović, M., Lulić, S., Moon, Y.-J. and Shanmugaraju, A. (2013) Propagation of Interplanetary Coronal Mass Ejections: The Drag Based Model. Solar Physics, 285, 295-315. https://doi.org/10.1007/s11207-012-0035-4

[3] Shanmugaraju, A. and Vršnak, B. (2014) Transit Time of Coronal Mass Ejections under Different Ambient Solar Wind Conditions. Solar Physics, 289, 339-349. https://doi.org/10.1007/s11207-013-0322-8

[4] Manoharan, P.K. (2006) Evolution of Coronal Mass Ejections in the Inner Heliosphere: A Study Using White-Light and Scintillation Images. Solar Physics, 235, 345-368. https://doi.org/10.1007/s11207-006-0100-y

[5] Manoharan, P.K., Gopalswamy, N., Yashiro, S., Lara, A., Michalek, G. and Howard, R.A. (2004) Influence of Coronal Mass Ejection Interaction on Propagation of Interplanetary Shocks. Journal of Geophysical Research: Space Physics, 109, A06109. https://doi.org/10.1029/2003JA010300

[6] Gopalswamy, N., Lara, A., Manoharan, P.K. and Howard, R.A. (2005) An Empirical Model to Predict the 1-AU Arrival of Interplanetary Shocks. Advances in Space Research, 36, 2289-2294. https://doi.org/10.1016/j.asr.2004.07.014

[7] Gopalswamy, N., Lara, A., Yashiro, S., Kaiser, M.L. and Howard, R.A. (2001) Predicting the 1-AU Arrival Times of Coronal Mass Ejections. Journal of Geophysical Research: Space Physics, 106, 29207-29217. https://doi.org/10.1029/2001JA000177

[8] Michalek, G., Gopalswamy, N., Lara, A. and Manoharan, P.K. (2004) Arrival Time of Halo Coronal Mass Ejections in the Vicinity of the Earth. Astronomy and Astrophysics, 423, 729-736.

[9] Kim, K.H., Moon, Y.J. and Cho, K.S. (2007) Prediction of the 1-AU Arrival Times of CME-Associated Interplanetary Shocks: Evaluation of an Empirical Interplanetary Shock Propagation Model. Journal of Geophysical Research: Space Physics, 112, A05104. https://doi.org/10.1029/2006JA011904 